\title{
Cavernous Hemangioma of the Adrenal Gland Mimicking Adrenal Cortical Carcinoma
}

\author{
Nurul N Jamaludin ${ }^{1}$, Rohaizak Muhammad ${ }^{2}$, Nurwahyuna Rosli ${ }^{3}$, Geok C Tan ${ }^{4}$, Shahrun NA Suhaimi ${ }^{5}$
}

\begin{abstract}
Cavernous hemangioma of the adrenal gland is a rare, non-functioning benign neoplasm. It is mostly a unilateral lesion and appears commonly at the age of 50 and 70 years. Most of the patients are asymptomatic and detected as incidentaloma. A 60-year-old man was incidentally found to have a right adrenal lesion during contrast-enhanced computed tomography (CECT) examination for chronic abdominal pain. The right adrenal lesion from the CECT abdomen measured $7 \mathrm{~cm}$ in diameter with features of adrenal carcinoma. Open right adrenalectomy was performed with the findings of $8 \mathrm{~cm}$ well-circumscribed right adrenal lesion with minimal adhesion to the inferior surface of the liver. The surgery was uneventful and the patient recovered well completely. The final histopathological examination confirmed a benign cavernous hemangioma. Adrenal cavernous hemangioma, which is a rare benign vascular malformation, might be difficult to be distinguished preoperatively from adrenal carcinoma because of the large size and similar features from computed tomography (CT) scan. Surgical excision is recommended due to the risk of spontaneous tumor rupture and the difficulty of ruling out malignancy.

Keywords: Adrenal, Adrenal carcinoma, Hemangioma, Incidentaloma.

World Journal of Endocrine Surgery (2020): 10.5005/jp-journals-10002-1293
\end{abstract}

\section{INTRODUCTION}

An adrenal incidentaloma is defined as an adrenal tumor which is detected on imaging performed for other indication. The incidence is about $5-10 \%{ }^{1}$ Most of the adrenal incidentaloma is $>1 \mathrm{~cm}$, without biochemical evidence or symptoms suggestive of cortical or medullary hypersecretion or general constitutional symptoms suggestive of malignant disease. The common causes are adenomas (80\%) followed by pheochromocytoma (5\%), malignant lesions, such as, adrenal cortical carcinoma (5\%), metastatic cancer from extra-adrenal primary (2\%), and other benign lesions including adrenal cyst, myelolipoma, hematoma, ganglioneuroma, and cavernous hemangioma. ${ }^{2}$ Adrenal cavernous hemangioma is a rare cause of adrenal incidentaloma and is mostly found incidentally on imaging studies. ${ }^{3}$ The clinical significance is that they can exhibit similar clinical and imaging features that are similar to adrenal cortical adenocarcinoma (ACC). ${ }^{4}$ On pathological inspection, cavernous hemangiomas of the adrenal gland involve the adrenal cortex and consist of multiple dilated vascular channels lined by a single layer of vascular endothelium surrounded by a collagenous wall. It is difficult to be distinguished clinically from ACC, thus surgery is strongly recommended. ${ }^{4}$

\section{Case Description}

A 60-year-old man presented with chronic left iliac fossa pain for 2 months. It was intermittent, colicky in nature, and self-limiting. There was no alteration of bowel habits, abdominal mass, or other alarming symptoms. He was hypertensive for 10 years and has been well controlled on a single antihypertensive, tablet amlodipine 10 mg daily. Physical examination was unremarkable.

A contrast-enhanced computed tomography (CECT) was done which showed a right $7.5 \times 6.5 \times 7 \mathrm{~cm}$ heterogeneous, hypodense lesion with a high-density rim of tissue at the periphery of the lesion. There were intralesional calcifications noted. It exhibited a delayed washout of contrast, $<60 \%$ on enhanced computed tomography (CT) scan. A left adrenal lesion was suggestive of an
${ }^{1}$ Department of Surgery, Faculty of Medicine and Health Sciences, Universiti Sains Islam, Negeri Sembilan, Malaysia

2,5Department of Surgery, Universiti Kebangsaan Malaysia, Kuala Lumpur, Malaysia

${ }^{3,4}$ Department of Pathology, Universiti Kebangsaan Malaysia, Kuala Lumpur, Kuala Lumpur, Malaysia

Corresponding Author: Rohaizak Muhammad, Department of Surgery, Universiti Kebangsaan Malaysia, Kuala Lumpur, Malaysia, Phone: +60 0193122555, e-mail: rohaizak@ppukm.ukm.edu.my

How to cite this article: Jamaludin NN, Muhammad R, Rosli N, et al. Cavernous Hemangioma of the Adrenal Gland Mimicking Adrenal Cortical Carcinoma. World J Endoc Surg 2020;12(2):93-95.

Source of support: Nil

Conflict of interest: None

angiomyolipoma (Fig. 1A). Biochemistry of serum electrolytes, dexamethasone suppression test, serum cortisol, plasma metanephrines and metanephrines, catecholamine, and 24-hour urinary catecholamine are all within the normal range.

Open right adrenalectomy with a preoperative diagnosis of right adrenal carcinoma was made. Intraoperative findings showed a $6 \times 8 \mathrm{~cm}$ well-circumscribed, encapsulated right adrenal mass with minimal adhesion to the inferior surface of the liver. There was neither tumor invasion to the surrounding structures nor the presence of metastatic features. It was removed en masse with an intact capsule. The surgery was uneventful and the patient recovered well without any surgical complication.

Histopathological examination revealed a well-circumscribed and encapsulated mass measuring $7 \times 6.6 \times 7 \mathrm{~cm}$. The mass has a central yellow-white cut surface with a friable and hemorrhagic periphery. Residual compressed adrenal tissue is noted at the periphery. Microscopically, the mass is composed of extensive necrotic and infarct-like center rimmed by large, dilated interconnected vascular channels lined by flattened endothelium 

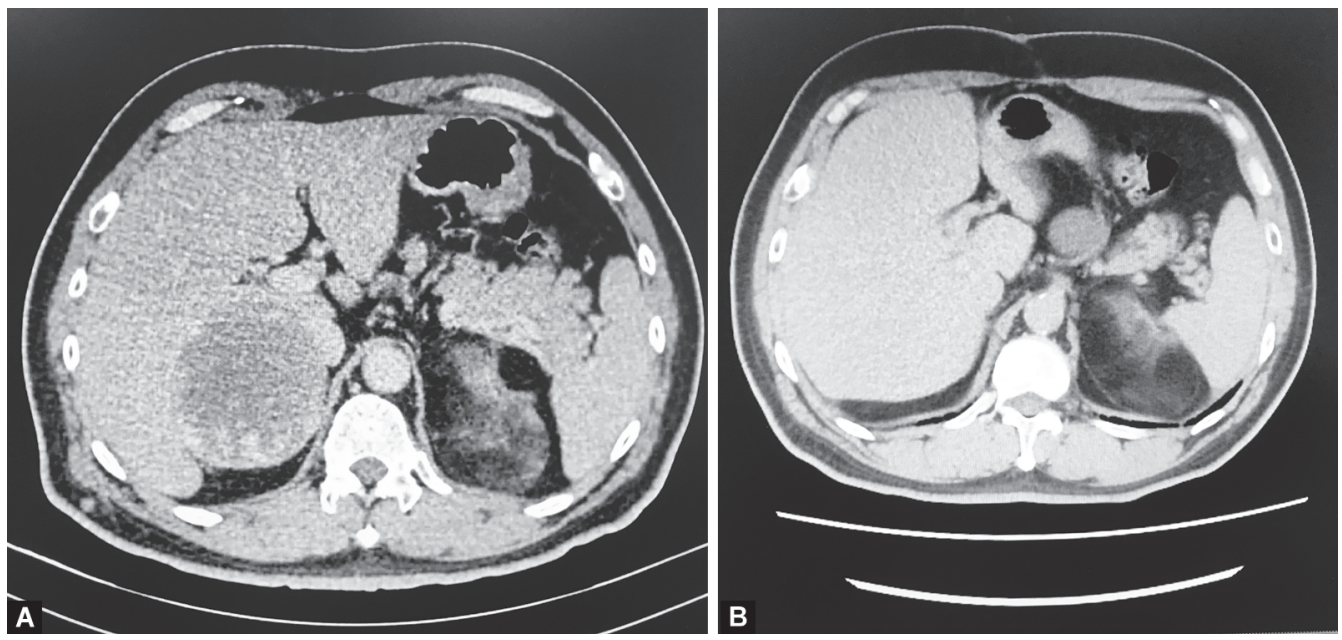

Figs $1 \mathrm{~A}$ and B: The CT scan shows bilateral adrenal mass before and after the surgery. The right adrenal mass was suspicious for malignancy with a diagnosis of left? angiomyolipoma
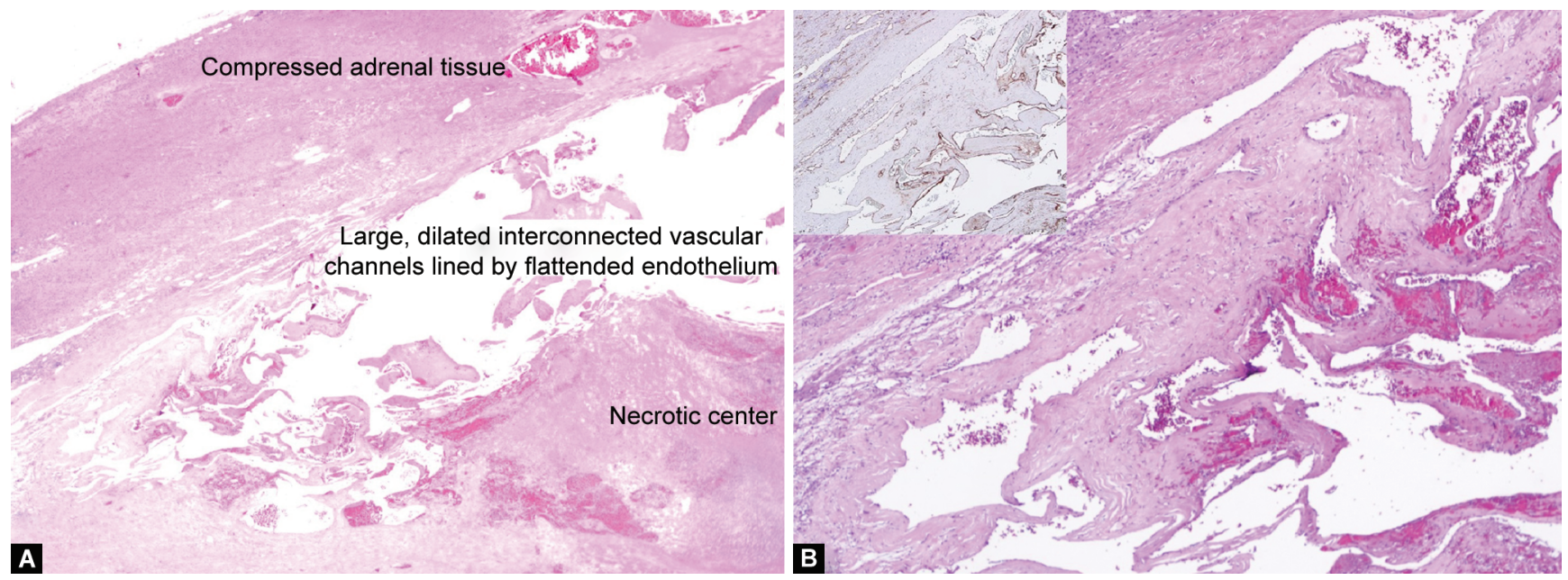

Figs 2A and B: (A) The mass is composed of an extensive necrotic center rimmed by large, dilated interconnected vascular channels lined by flattened endothelium. Adjacent to the mass is adrenal tissue which is compressed (H\&E, $\times 20)$; (B) With a higher magnification of the vascular channels, some are engorged with red blood cells $(\mathrm{H} \& \mathrm{E}, \times 40)$. The endothelial cells are highlighted by CD31 immunostain (inset)

(Fig. 2A). Some of the vessels are engorged with red blood cells. The vascular spaces are lined by endothelial cells which are positive for CD31 (Fig. 2B). In areas, the periphery of the tumor is fibrotic with scattered blood vessels. A diagnosis of cavernous hemangioma of the adrenal was made. A repeat CT scan was done at 3 months after surgery and the patient was well without evidence of recurrence (Fig. 1B).

\section{Discussion}

Adrenal cavernous hemangiomas are extremely rare. There are only a few cases have been reported in the literature. ${ }^{3}$ Most of them are unilateral and appear commonly between the age of 50 and 70 years, with male to female ratio of 1:2. Most of the patients are asymptomatic and detected as incidentaloma, as in our patient. Some might present with abdominal pain or even hypovolemic shock due to spontaneous tumor rupture. ${ }^{1}$ The majority of them are non-functioning tumors, but three cases of hormone-secreting adrenal hemangiomas had been reported to date., ${ }^{1,5,6}$

Ultrasound is difficult to differentiate cavernous hemangiomas from other adrenal lesions. Although CT is helpful, however, it may be difficult to distinguish it from malignant adrenal tumors. ${ }^{5,7}$ The suspicious findings of adrenal cavernous hemangioma on CT include a hypodense, heterogeneous lesion with calcifications, as seen in the patient. Characteristic calcifications have been reported in $28-87 \%$ of cases and described as speckled appearance throughout the entire mass, or centrally located with an irregular, stellate branching pattern or even some peripheral crescentic calcifications. These represent phleboliths within the dilated vascular spaces of the lesion. ${ }^{7}$ However, this patient does not have the pathognomonic irregular stellate pattern on CT to suggest a cavernous hemangioma. Cavernous hemangiomas also tend to exhibit delayed washout of CECT as seen in ACC. ${ }^{8}$ Therefore, it is difficult to be distinguished from ACC. In this patient, the absence of enhancement in CECT is probably due to the presence of extensive necrosis of the adrenal which obscured the entrance of the contrast agent into the mass.

Large adrenal incidentaloma which is $>4 \mathrm{~m}$ is more likely to be malignant. ${ }^{9}$ The characteristic of the lesion which was large, $7 \times 6.5 \times 7 \mathrm{~cm}$ with CT scan features of calcification, high Hounsfield unit (30), delayed washout of contrast even in the absence of local 
invasion or metastasis seen in this patient, made it likely to be adrenal carcinoma until proven otherwise.

Surgical excision is the mainstay of treatment for this patient for diagnostic uncertainty of malignancy. The role of laparoscopic adrenalectomy in the case of suspicion of malignancy is debatable. Some retrospective studies on the treatment of adrenocortical carcinoma show no difference in terms of recurrence rate, time to recurrence, or overall survival between laparoscopic and open surgery. ${ }^{10-12} \mathrm{~A}$ few studies had the opposite detrimental result with higher rates of peritoneal carcinomatosis, increased recurrence rates, the incompleteness of resection, and lowered overall survival. ${ }^{13,14}$ Given this, we decided to perform open adrenalectomy because of suspicion of malignancy.

\section{Conclusion}

Adrenal cavernous hemangiomas are rare benign tumors that might be difficult to be distinguished from adrenocortical carcinoma, due to lack of specificity in preoperative studies. Surgical excision is recommended due to the risk of spontaneous tumor rupture and the difficulty in ruling out malignancy.

\section{References}

1. Edwards JP, Stuart HC, Urbanski SJ, et al. A rare cavernous hemangioma of the adrenal gland. Int J Surg Case Rep 2014;5(2):5255. DOI: 10.1016/j.ijscr.2013.11.003.

2. Terzolo M, Stigliano A, Chiodini I, et al. AME position statement on adrenal incidentaloma. Eur J Endocrinol 2011;164(6):851-870. DOI: 10.1530/EJE-10-1147.

3. Nishtala M, Cai D, Baughman W, et al. Adrenal cavernous hemangioma: a rare tumor that mimics adrenal cortical carcinoma. Surgery Open Science 2019;1(1):7-13. DOI: 10.1016/j.sopen.2019.04.001.

4. Auh $\mathrm{YH}$, Anand J, Zirinsky K, et al. Adrenal hemangioma: a case report. J Comput Tomogr 1986;10(1):57-59. DOI: 10.1016/0149936X(86)90012-3.
5. Telem DA, Nguyen SQ, Chin EH, et al. Laparoscopic resection of giant adrenal cavernous hemangioma. JSLS 2009;13(4):260-262. DOI: 10.4 293/108680809X12589999538110.

6. Sabanegh Jr E, Harris MJ, Grider D. Cavernous adrenal haemangioma. Urology 1993;42(3):327-330. DOI: 10.1016/0090-4295(93) 90626-L.

7. Boraschi P, Campatelli A, Di Vito A, et al. Hemorrhage in cavernous hemangioma of the adrenal gland: US, CT and MRI appearances with pathologic correlation. Eur J Radiol 1995;21(1):41-43. DOI: 10.1016/0720-048X(96)81068-0.

8. Lee WJ, Weinreb J, Kumari S, et al. Case report. adrenal hemangioma. J Comput Assist Tomogr 1982;6(2):392-394. DOI: 10.1097/00004728198204000-00028.

9. Ballian N, Adler JT, Sippel RS, et al. Revisiting adrenal mass size as an indication for adrenalectomy. J Surg Res 2009;156(1):16-20. DOI: 10.1016/j.jss.2009.03.071.

10. Lombardi CP, Raffaelli M, De Crea C, et al. Open versus endoscopic adrenalectomy in the treatment of localized (stage I/II) adrenocortical carcinoma: results of a multi-institutional Italian survey. Surgery 2012;152(6):1158-1164. DOI: 10.1016/j.surg.2012.08.014.

11. Porpiglia F, Fiori C, Daffara F, et al. Retrospective evaluation of the outcome of open versus laparoscopic adrenalectomy for stage I and II adrenocortical cancer. Eur Urol 2010;57(5):873-878. DOI: 10.1016/ j.eururo.2010.01.036.

12. Brix D, Allolio B, Fenske W, et al. Laparoscopic versus open adrenalectomy for adrenocortical carcinoma: surgical and oncologic outcome in 152 patients. Eur Urol 2010;58(4):609-615. DOI: 10.1016/j. eururo.2010.06.024.

13. Leboulleux S, Deandreis D, Al Ghuzlan A, et al. Adrenocortical carcinoma: is the surgical approach a risk factor of peritoneal carcinomatosis? Eur J Endocrinol 2010;162(6):1147-1153. DOI: 10.1530/ EJE-09-1096.

14. Miller BS, Gauger PG, Hammer GD, et al. Resection of adrenocortical carcinoma is less complete and local recurrence occurs sooner and more often after laparoscopic adrenalectomy than after open adrenalectomy. Surgery 2012;152(6):1150-1157. DOI: 10.1016/ j.surg.2012.08.024. 